\title{
A Video-based Cargo Fire Verification System for Commercial Aircraft: Design and Test Methods
}

\author{
WOLFGANG KRÜLL ${ }^{1}$, INGOLF WILLMS ${ }^{1}$, RADOSLAW R. ZAKRZEWSKI ${ }^{2}$, \\ MOKHTAR SADOK ${ }^{2}$, JEFF SHIRER ${ }^{2}$, and BOB ZELIFF ${ }^{2}$ \\ ${ }^{1}$ Department of Communication Systems NTS, University Duisburg-Essen, \\ Duisburg, Germany \\ ${ }^{2}$ Goodrich Corporation, Vergennes, Vermont, U.S.A.
}

\begin{abstract}
The Cargo Fire Verification System was developed to address the problem of frequent false smoke alarms that are of particular concern in long range flights of passenger aircraft. The system uses low-cost CCD cameras operating in the near infrared range to directly detect fire and hotspots. In addition, LED illumination units are appropriately switched on and off, and the obtained images are analysed to detect smoke. Fusion of image processing results with temperature and humidity readings allows reliable detection of true fires and elimination of false alarms due to condensed moisture (fog) and dust. It was necessary to create a suite of fire sensitivity and false alarm immunity tests applicable to these vision based fire detection systems. This paper will concentrate on the design of the system, testing aspects, and test cell modifications.
\end{abstract}

KEYWORDS: fire detection, aircraft, cargo bay, cargo compartment, smoke detection, false alarms, dust, humidity, test methods, video-based, detection, CCD

\section{INSTRUCTION}

Development of the Cargo Fire Verification System (CFVS) was motivated by the need to reduce the incidence of false alarms of conventional smoke detection systems used in cargo bays of commercial aircraft. Upon an alarm, the crew is typically required to release fire suppressant and to divert to the nearest airport. Each emergency landing due to a false alarm incurs significant cost to the air carrier. In addition, diversion to a small remote airport may itself pose significant danger to the passengers or the aircraft. In case of long range flights over polar regions, the nearest airport may lack the necessary facilities, so safe take-off may be questionable in harsh weather environment. Using only a conventional system, the pilot has no capability to verify if the alarm is real or false. The very short detection time of less than 60 seconds [1,2] is responsible for the high ratio of false alarms to the detection of fires. For the period 1995 - 1999, the ratio was approximately 200:1 [3].

Possible reasons for false alarms of traditional smoke detectors are mist, dust, and oil particles. Additional problems in cargo compartments are environmental conditions such as temperature and air pressure variations. The objectives of the Cargo Fire Verification System are to provide the aircrew with images of the conditions in the cargo bay, to detect fire earlier than conventional smoke detectors, and to greatly reduce false alarms. Cargo compartments of passenger aircraft are located below the passenger cabin and are not easily accessible during flight. They are a difficult area to view with video cameras because the gap between the cargo and the bay ceiling can be as small as $4.3 \mathrm{~cm}$. For reference, the size of an Airbus A340-500 aft compartment is $10.4 \mathrm{~m}$ long, $4.2 \mathrm{~m}$ wide, and $1.7 \mathrm{~m}$ high. The A340-500 contains 3 cargo compartments, referred to as the Forward (in front of the aircraft), Aft, and Bulk bays (behind the wing boxes). 


\section{SYSTEM ARCHITECTURE}

The core of the CFVS are CCD cameras operating in the near infrared (NIR) band. Two cameras are located in opposite corners of each cargo bay, providing full visibility of the entire bay. In order to make them immune to external illumination sources, the cameras use optical filters that block visible light. Typically, cargo bays are equipped with fluorescent lighting with no emissions in the NIR band. Therefore, the CFVS operation does not depend on whether the cargo bay light is on or off. Each camera is equipped with its own controlled NIR LED illumination source. Additional NIR illumination units are installed in the ceiling of the bay. Through appropriate switching of those illumination sources, the system obtains different views of the scene, as described in a following section. An example of placement of cameras and overhead illumination sources in A340 cargo bays is shown in Fig. 1.

Each camera is equipped with a DSP processor to analyse captured images and to calculate their various numerical features, which are then sent to the central control module. Additionally, the CFVS collects temperature and humidity measurements from sensors placed within cameras and overhead illumination units. These values are used to assess possibility of a false alarm-inducing scenario such as ascent-related fog. The central processing unit analyses video and non-video data and upon an alarm issued by the primary system produces confirmed or unconfirmed diagnosis, together with the appropriate highlighting of the images sent to the cockpit video display.
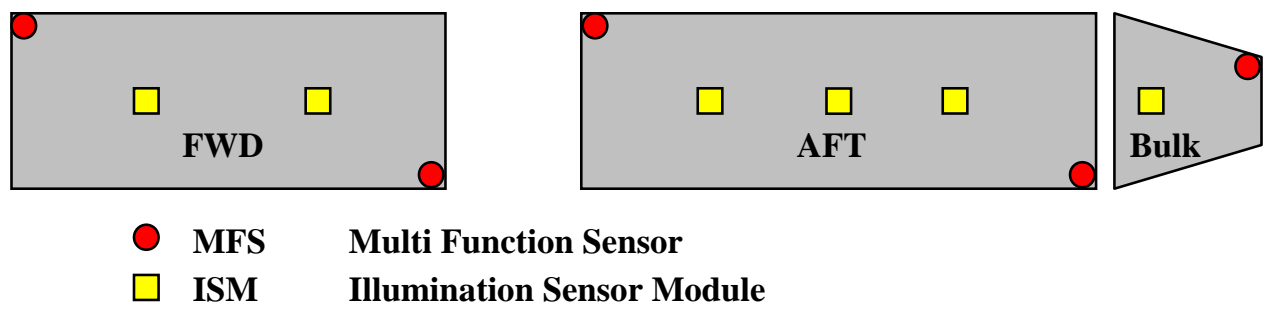

Fig. 1. Example CFVS aircraft installation, top view.

In addition to decision making, the central unit acts as a digital video recorder. Output of each camera is recorded at a lower frame rate, so that it can be viewed at any time at the push of a button. This allows the crew to examine the state of a cargo bay before and after a primary alarm. In the present implementation, the CFVS stores the most recent 10 minutes of video from each camera. With addition of memory chips or adjustment of recorded frame rate, the length of this time window may be modified as needed.

\section{Light Switching Sequences}

To detect various visual aspects of fire and smoke and to differentiate it from non-fire aerosols such as fog and dust, the CFVS analyses different views obtained under different illumination conditions. In the current implementation, four distinct views are used. Figure 2 shows examples of these four views through a simulated $4.3 \mathrm{~cm}$ gap above a container, acquired in University of Duisburg-Essen Fire Detection Laboratory.

In the dark view, all illumination sources are turned off, so that the presence of any high intensity image areas indicates a heat source, as illustrated in the upper-left part of Fig. 2. This view is used for flame and hotspot detection, which is sufficient in majority of fire cases. However, in a fully loaded cargo bay, flames may be hidden behind containers. Similarly, for smouldering fires similar to EN54 fires [7] TF2 and TF3 no flames may be 
visible. In such cases, the system must use the alternate smoke detection mode. For this, the remaining three illuminated views are used. In the overhead view, the cameras' LED sources are switched off, while the overhead illumination units are turned on. In presence of smoke, their light is scattered, resulting in brighter image areas, as illustrated in the upper-right part of Fig. 2. In many cases, the overhead light provides quickest smoke detection.

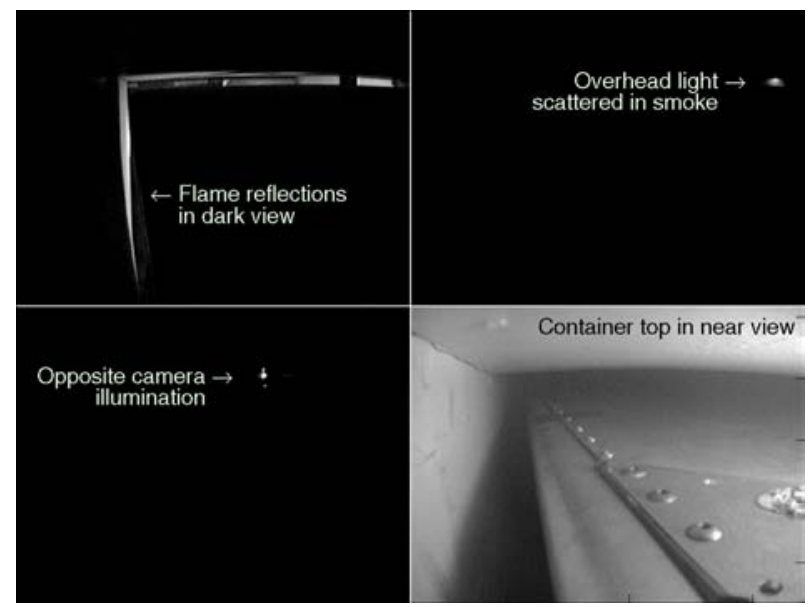

Fig. 2. Four views with different illumination, same geometric configuration.

In the opposite view, all illumination sources are turned off, except for the LED of the opposite camera. In absence of smoke, the opposite light is well visible, as seen in the lower-left part of Fig. 2. With smoke, the light gets absorbed and the image becomes smaller and dimmer until it may disappear completely. It may be noted that the CFVS operating in the opposite view mode acts as a very long optical smoke detector, whose length comprises the entire bay. A pair of cameras placed on opposite ends of the bay replaces, and offers detection superior to, an entire set of multiple conventional point smoke detectors. For this mode to work, however, there must be a clear gap between the top of the cargo (containers) and the ceiling, e.g., as stated in Airbus loadability conditions. Presence of any cargo obstructing visibility of the opposite light may make this view useless from the detection point of view. However, the system may analyse such situation prior to take-off and switch to one of the other detection modes.

The last of the illuminated views is the so-called near view, in which the only light turned on is the one collocated with the camera that is acquiring images. It is the near view that is fed to the central unit and recorded for later use by the crew. An example of a near view image is shown in the lower-right portion of Fig. 2. Smoke may be visible in the near view through scattering of light by an aerosol cloud.

\section{Performance Requirements}

The main challenge in design of the CFVS image processing and decision making algorithms was to specify clear and verifiable performance criteria. On one hand, the CFVS must always detect true fire or smoke prior to the primary system, so that when the latter issues its alarm the CFVS is ready with a confirmation decision. On the other hand, the common false alarm scenarios should be recognized as such. This required detailed characterization of the most common false alarm causes. 
We used available public domain studies such as [4], as well as proprietary false alarm statistics provided by Airbus. In addition, an advisory group of university and industrial experts was formed and asked to determine which false alarm scenarios are prevalent in practice and may be successfully distinguished from true smoke or fire. A conclusion of this study was that the overwhelming majority of false alarms is most likely caused by fog. For example, fog may form through super-saturation of humid air due to rapid pressure decrease and adiabatic cooling during take-off and ascent. Fog may also form during the flight near moisture-emitting cargo such as vegetables or animals.

The second most probable false alarm cause was determined to be dust lifted from dirty containers, or perhaps produced by cargo such as pollinating plants or agitated animals. The key to fog and dust differentiation from smoke is appropriate choice of the image features that change more in fog or dust than in smoke [5]. Feature selection was based on experimental video database that included fire, fog and dust events. Image features associated with smoke are chosen in such a way that they are not masked by false alarm causes such as dust or fog, so that if a fire occurs when fog is present it will still be detected and an alarm will be issued as expected.

\section{DETECTION AND DISCRIMINATION ALGORITHMS}

Image processing is performed by DSP chips located within each camera. It is done on a single frame basis - i.e., each frame is analysed independently. Then the calculated values of image features are sent over a low-bandwidth digital link to the control unit which then performs data fusion and makes a fire/non-fire decision.

Different image processing algorithms are used for the four different views. To save processor cycles, priority was given to simple image features well known in literature [6]. In some views, only suitably selected image windows are processed to amplify the analyzed effects and to further reduce computational requirements.

In what follows, $X(i, j)$ denotes pixel intensity, with $i$ and $j$ being coordinates within the image or its window of dimension $M$ by $N$. Then, the two particularly useful image features are mean pixel intensity and its standard deviation, which can be found as

$$
\begin{aligned}
& \operatorname{mean}(X)=\frac{1}{M N} \sum_{i=1}^{M} \sum_{j=1}^{N} X(i, j) \\
& \operatorname{stdev}(X)=\sqrt{\frac{1}{M N} \sum_{i=1}^{M} \sum_{j=1}^{N}(X(i, j)-\text { mean }(X))^{2}}
\end{aligned}
$$

These two image statistics provide useful information about global intensity level and its variability, but do not allow any inference about its spatial distribution. This may be achieved by analysing the following three second order moments

$$
\begin{aligned}
& \mathrm{M}_{20}(X)=\frac{1}{M N} \sum_{i=1}^{M} \sum_{j=1}^{N}\left(i-\mathrm{X}_{c}\right)^{2} X(i, j) \\
& \mathrm{M}_{02}(X)=\frac{1}{M N} \sum_{i=1}^{M} \sum_{j=1}^{N}\left(j-\mathrm{Y}_{c}\right)^{2} X(i, j) \\
& \mathrm{M}_{11}(X)=\frac{1}{M N} \sum_{i=1}^{M} \sum_{j=1}^{N}\left(i-\mathrm{X}_{c}\right)\left(j-\mathrm{Y}_{c}\right) X(i, j)
\end{aligned}
$$

where $\mathrm{X}_{c}$ and $\mathrm{Y}_{c}$ are the center of mass coordinates for the intensity field, given by 


$$
\mathrm{X}_{c}(X)=\frac{1}{M N} \sum_{i=1}^{M} \sum_{j=1}^{N} i X(i, j) \quad \mathrm{Y}_{c}(X)=\frac{1}{M N} \sum_{i=1}^{M} \sum_{j=1}^{N} j X(i, j)
$$

The above calculations may be applied to raw images, as captured by the camera, or after suitable image transformations. For example, to characterize changes in image sharpness, mean value, standard deviation or the second order moments $\mathrm{M}_{20}, \mathrm{M}_{02}$, and $\mathrm{M}_{11}$ may be applied to the transformed gradient norm image $G_{N}$ given by

$$
\begin{aligned}
& G_{1}(i, j)=X(i+1, j)-X(i, j) \\
& G_{2}(i, j)=X(i, j+1)-X(i, j) \\
& G_{N}(i, j)=\sqrt{G_{1}{ }^{2}(i, j)+G_{2}{ }^{2}(i, j)}
\end{aligned}
$$

For hotspot analysis we applied standard image segmentation to detect connected, spaceand time-wise, regions of elevated intensity. To reduce influence of noise effects on detection, each feature is appropriately filtered. The simplest first-order filter is

$$
y_{\text {filt }}(t)=(1-\alpha) y_{\text {filt }}(t-\Delta)+\alpha y(t)
$$

where $\alpha$ is the filtering constant, $\Delta$ is the time interval between image acquisitions and $y(t)$ and $y_{\text {filt }}(t)$ are the raw and filtered feature values. The filtered values are then sent to the central processing unit, which compares them against detection thresholds [5] and performs final decision making. In a simplest form, a decision function may involve checking whether a filtered image feature crossed its associated threshold

$$
D_{i}=\left(y_{\text {filt } i}>t_{i}\right)
$$

A more complicated form may involve a linear combination of image features

$$
D_{i}=\left(\sum_{j=1}^{k} a_{j} y_{\text {filt } j}>t_{i}\right)
$$

Decision functions $D_{i}$ may also involve non-image measurements such as temperature, pressure, or humidity. The final decision may be a Boolean function of multiple elementary decisions $D_{i}$. Other more involved decision making methods may also be used, such as fuzzy logic. They fuse image data with non-image measurements such as temperature, humidity or flight phase status to obtain the best decision [5]. The choice of the particular image features and decision functions is based on experimental video database. While certain guidance may be gathered from the general nature of the observed images, such as fading, loss of contrast, or light blooming, the final selection can only be made based on the actual video data. The decision functions and their thresholds are chosen in such a way so that correct classification of all true fire cases is always assured, but the incidence of false alarms is minimized.

\section{TESTING OF VIDEO-BASED SYSTEMS}

The existing testing guidelines and standards for qualification of current smoke detection systems were formulated mainly to address the case of traditional detection technologies, e.g., optical-based smoke detectors. In many cases, the qualification tests were derived from the EN-54 [7] fire sensitivity tests, which were originally developed for testing smoke detectors in buildings. In short, the EN-54 tests have different types of smoke sources at the center of the floor of a large test chamber. During the tests, the smoke 
density at the location of the smoke detector and the alarm time of the detector are measured. The geometry and contents of the test chamber are not critical to the smoke detectors if the smoke density increases within the EN-54 guidelines. However, for a video-based detection system, the scene geometry and contents are critical. The scene should closely represent the actual application. For an aircraft cargo bay application, the fire test room should resemble an actual cargo bay, including cargo obstructions in the camera field of view. Tests should also retain the repeatability aspects of the EN-54 tests.

Possible candidates for the testing of the CFVS included special testing aircraft, a reconstruction of a cargo compartment, and a modified EN-54 test room. Testing in an aircraft has many of disadvantages, e.g., high costs, limited test times, and restricted test options (no open fires and dust tests are permitted). Figure 3 is an image from a video recording of a smoke test in an A340-600 aircraft.

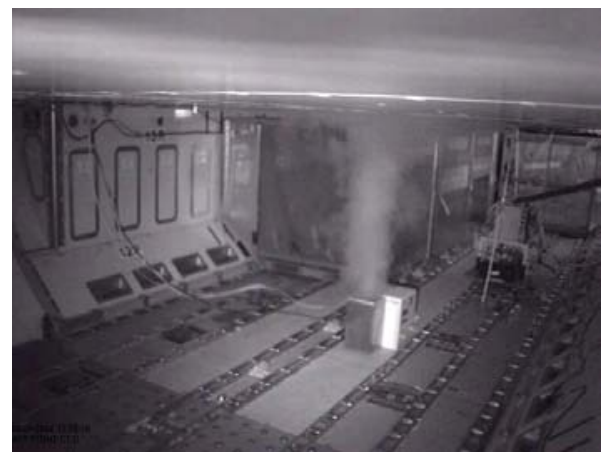

Fig. 3. Smoke generator test in an A340-600 aircraft.

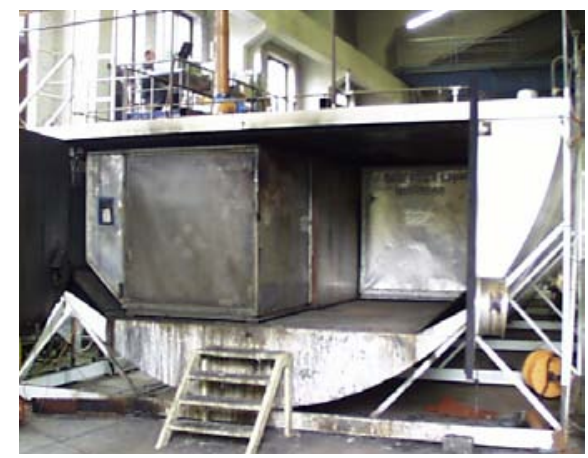

Fig. 4. A340-500 cargo bay mock-up in Trauen, Germany.

Tests in a reconstruction of a cargo compartment, such as the A340-500 cargo bay mockup in Trauen, Germany, have the advantage that the geometry is very close to the actual cargo bay, Fig. 4 shows the mock-up. The main drawbacks of the Trauen mock-up are the difficult adaptation to a special compartment type, high construction effort, and the required space only for this application. Furthermore, no comparative EN-54 tests are possible because of the smaller volume of the mock-up, the uncontrollable environmental conditions, and the lack of required instrumentation. Because of the disadvantages listed above, the tests were performed in the fire detection lab of the University DuisburgEssen, which is traditionally used for EN-54 tests.

\section{Fire Room Configuration}

The Duisburg fire lab exhibits an EN-54 like test cell with the option of changing the height of the cell by moving the ceiling in a wide range. Advantages of the fire lab are controlled environmental conditions, a wide range of measurement possibilities, and a significantly better probability for "repeatable" tests than other testing options. The fire room was modified so that it more closely resembled a cargo bay in the following ways (see Fig. 5, and Fig. 10):

- Aluminium side wall plates were hung down $95 \mathrm{~cm}$ from the ceiling at a distance of $210 \mathrm{~cm}$ from the centerline to reduce the room width to that of a cargo compartment, emulating a cargo bay width of $420 \mathrm{~cm}$. To enhance the realism of the side walls, the plates were covered with DuPont Tedlar PVF Film as in an actual cargo bay. 
- A single $1 \mathrm{~m}^{2}$ sheet metal plate covered with the cargo bay lining material was mounted on the ceiling near the cameras in order to provide realistic optical conditions near the cameras.

- Fire and non-fire sources were placed on a platform, so that the distance between the source and the fire room ceiling was $1.7 \mathrm{~m}$, which is the floor to ceiling distance in a cargo bay. A platform was required to raise the source because the ceiling cannot be lowered closer than $2.87 \mathrm{~m}$ from the fire room floor.

- LD-3 cargo containers were placed in front of the cameras to limit the field of view to the gap between the top of the container and the ceiling. Both containers were set on platforms (see Fig. 5 and Fig. 6) so that the gap between the container and the ceiling was in the worst case at a minimum value of $4.3 \mathrm{~cm}$. The containers were located according to existing cargo loading standards.
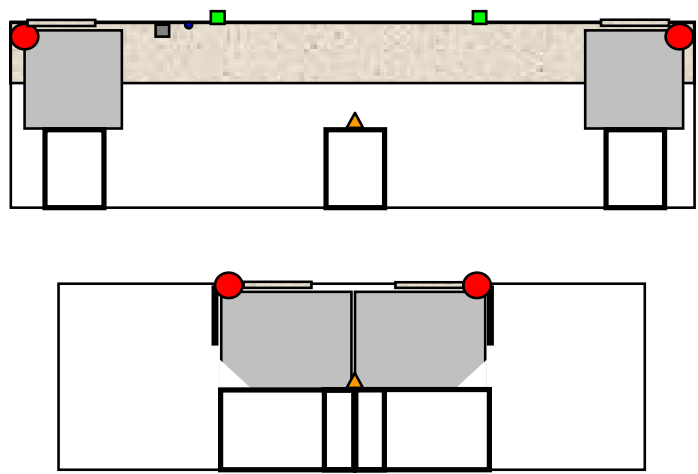

Fig. 5. Modified Duisburg test cell side view and front view.

\section{Fire Tests}

A suite of fire sensitivity tests applicable to vision based fire detection systems was then developed by Goodrich Corporation and the Universität Duisburg-Essen. The objective was to scale the standard EN-54 fire sensitivity tests $[7,8]$ for the reduced volume of the modified test cell as described above. Fire and smoke tests were performed in the A340500 cargo bay mock-up in Trauen and in Duisburg. At the Duisburg fire lab tests were performed in three major types of scenarios, for details see Table 1:

- $\quad$ Standard EN-54 test fires.

- Modified test fires without test cell modifications, but with camera obstructions by containers.

- Modified test fires with test cell modifications (for Forward, Aft, and Bulk compartment configurations), including camera obstructions by containers. The test cell was modified to more closely resemble an actual cargo compartment, and the test fire material was reduced to account for the reduced test cell volume.

Even though the room geometries of both test cells (the reconstruction of a cargo compartment in Trauen and the Duisburg fire lab) are completely different, the results are comparable by using a simple scaling factor. If smoke density (via the extinction measuring device, MIREX [7]) over time values measured in Duisburg (without side walls) are multiplied with a factor "3" the values are comparable with Trauen results [9]. 
Table 1. EN-54 and modified test fires.

\begin{tabular}{|c|c|c|c|c|c|}
\hline & & \multicolumn{2}{|c|}{ Modified test fire } & \multicolumn{2}{c|}{ EN 54 test fire } \\
\hline TF & Material & Amount & Pan size & Amount & Pan size \\
\hline 1 & beech wood & 40 pieces & - & 72 pieces & \\
\hline 2 & beech wood & 16 pieces & - & 30 pieces & \\
\hline 3 & cotton & 54 wicks & - & 108 wicks & \\
\hline 4 & polyurethane & 2 mats & - & 4 mats & \\
\hline 5 & n-heptane & $120 \mathrm{~g}$ & $12 \mathrm{~cm}^{*} 12 \mathrm{~cm} * 2 \mathrm{~cm}$ & $650 \mathrm{~g}$ & $33 \mathrm{~cm}^{*} 33 \mathrm{~cm}^{*} 5 \mathrm{~cm}$ \\
\hline 6 & alcohol & $850 \mathrm{~g}$ & $33 \mathrm{~cm}^{*} 33 \mathrm{~cm}^{*} \mathrm{~cm}$ & $2300 \mathrm{~g}$ & $50 \mathrm{~cm}^{*} 5 \mathrm{~cm}^{*} 5 \mathrm{~cm}$ \\
\hline 7 & decalene & $75 \mathrm{~g}$ & $10 \mathrm{~cm}^{*} 10 \mathrm{~cm} * 2 \mathrm{~cm}$ & $170 \mathrm{~g}$ & $12 \mathrm{~cm}^{*} 12 \mathrm{~cm}^{*} 2 \mathrm{~cm}$ \\
\hline
\end{tabular}

The Duisburg test cell volume was $293 \mathrm{~m}^{3}(10.5 \mathrm{~m} * 9.0 \mathrm{~m} * 3.1 \mathrm{~m})$ and the Trauen mock-up volume was $103 \mathrm{~m}^{3}(15.0 \mathrm{~m} * 4.2 \mathrm{~m} * 1.71 \mathrm{~m})$, approx. factor "3" between both rooms. Figure 6 compares the Duisburg test cell and Trauen mock-up (smaller area, more containers). Figure 7 compares smoke density values for TF2 runs at both locations and scaled values.
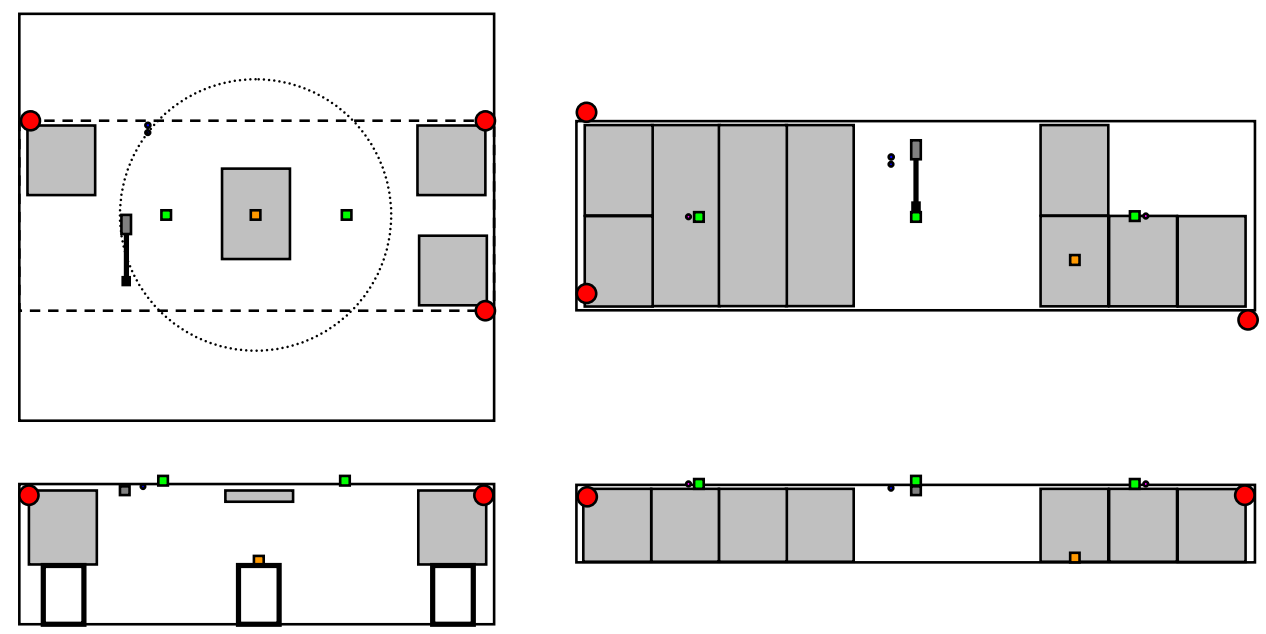

Fig. 6. Comparison of Duisburg top view (upper left), Trauen top view (upper right), Duisburg side view (lower left), and Trauen side view (lower right).

Further tests with side walls in Duisburg show very good results with respect to the reconstruction of a cargo compartment in the Trauen - it is still a simple scaling factor to approximate the results from Trauen. A scaling factor of "1.5" shows a good approach to Trauen data bay (see Fig. 8).

Traditional smoke detectors were also part of the preliminary scaling experiments, and the smoke densities at which they alarmed were determined for each test fire type. The CFVS was required to detect the test fires at a lower smoke density than the traditional smoke detectors. Figure 9 shows a typical example of CFVS performance compared to actual behaviour of production A340 smoke detectors. The decision function shown is based on analysis of the overhead view. For a smouldering fire, the CFVS is ready to confirm almost half a minute prior to the conventional smoke alarm. 


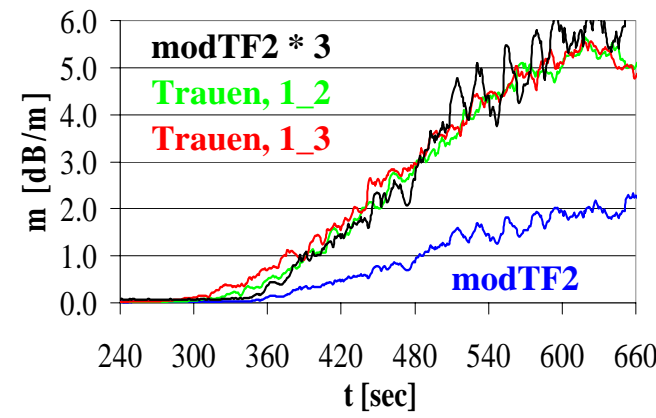

Fig. 7. MIREX values of modified TF2, measured in Trauen and Duisburg (without side walls), scaled values.

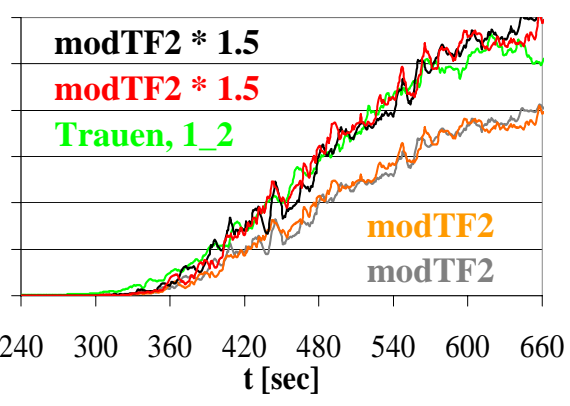

Fig. 8. MIREX values of modified TF2 measured in Trauen and Duisburg (with side walls), scaled values.

\section{Dust Tests}

In addition to fire tests, non-fire tests with dust were set-up and performed. Dust is known to cause problems with optical smoke detectors and not only in cargo bays. Depending on the dust type and the dust production it may look similar to smoke on video. Thus, the discrimination of dust and smoke in video-based systems is a challenge in its own. Therefore special consideration was given to dust tests and measures in order to provide a high degree of repeatability.

Modified TF2 fire; CFVS detects fire 29s before conventional smoke alarm

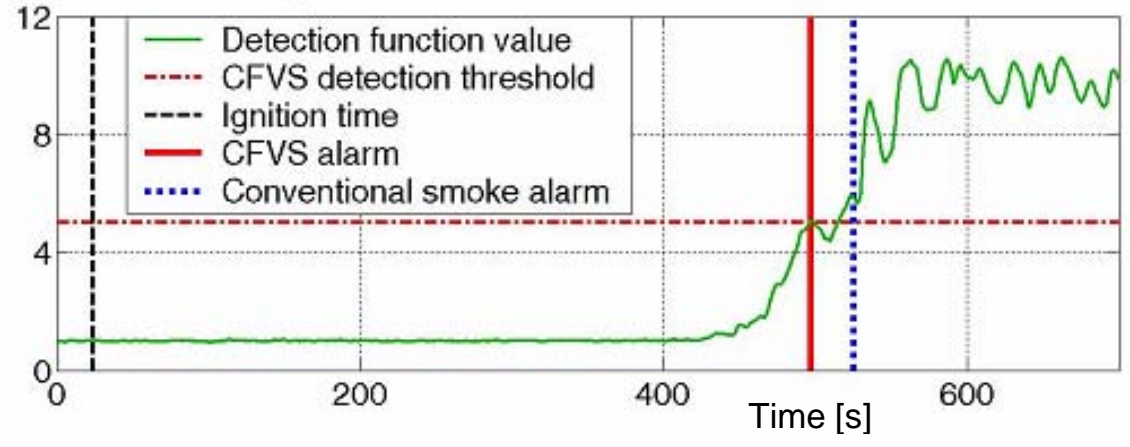

Fig. 9. Modified TF2 fire with alarm times.

The dust generator was placed on a platform in the center of the test cell, in the same location as the test fire source. LD-3 cargo containers on platforms limited the view of the video cameras to the $4.3 \mathrm{~cm}$ gap between the containers and the ceiling. In bulk bay configuration, the dust generator was moved closer to the lone video camera in response to the smaller size of the Bulk compartment. Figure 11 shows the set-up for the dust test. The key factors in the dust tests included the type of dust, the rate of dust introduced into the test cell, and amount of time that dust was generated, and the location of the dust generator.

Dust is typically defined as "small solid particles, conventionally taken as those particles below $75 \mu \mathrm{m}$ in diameter, which settle out under their own weight but which may remain suspended for some time" [10]. Standardized dust particles are designated in several 
groups [11]. Two of them, A1 dust (Ultrafine, particles size 0-10 $\mu \mathrm{m}$ ) and A2 dust (Fine, particles size 0-80 $\mu \mathrm{m}$ ), were used for testing in Duisburg.

The small particles in these dusts remain airborne longer than large particles and are more likely to be detected as smoke. They also are close to the particle sizes found in wood smoke, which makes it difficult for detection systems to distinguish between smoke and dust.

A dust generator was used to introduce dust into the test chamber. The generator adds the dust to a compressed air stream which is then directed by a nozzle into the test cell. The dust was expelled at a constant rate of $140 \mathrm{~g} / \mathrm{s}$. The dust nozzle was directed vertically towards the ceiling. By adjusting the compressed air pressure and the height of the dust generator, it was possible to create a dust cloud just below the ceiling. This put the dust cloud directly into the CFVS field of view. This type of dust generation is also analogous to the smoke generation of the test fires described above. In the test fires, the smoke rises in a vertical stream and then forms a cloud beneath the ceiling.

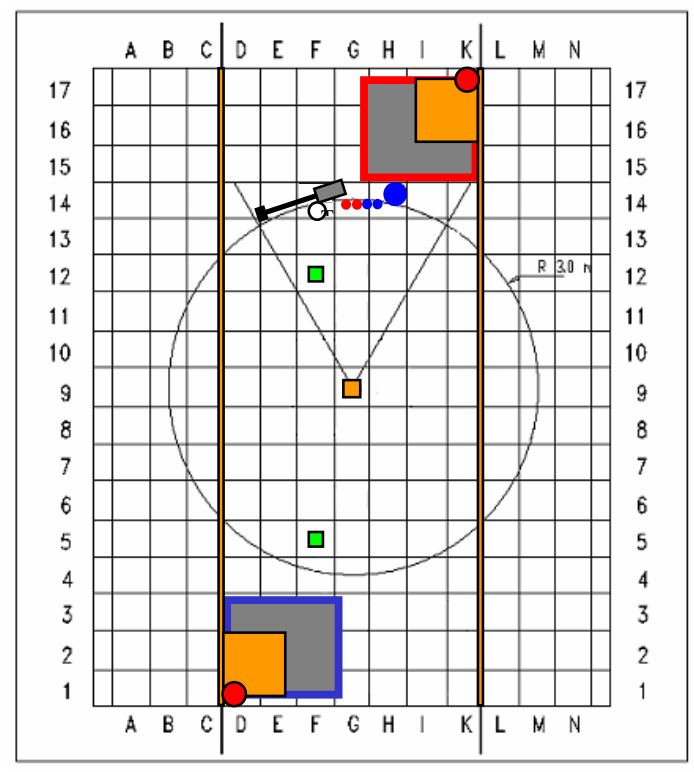

Fig. 10. Modified Duisburg test cell, top view.

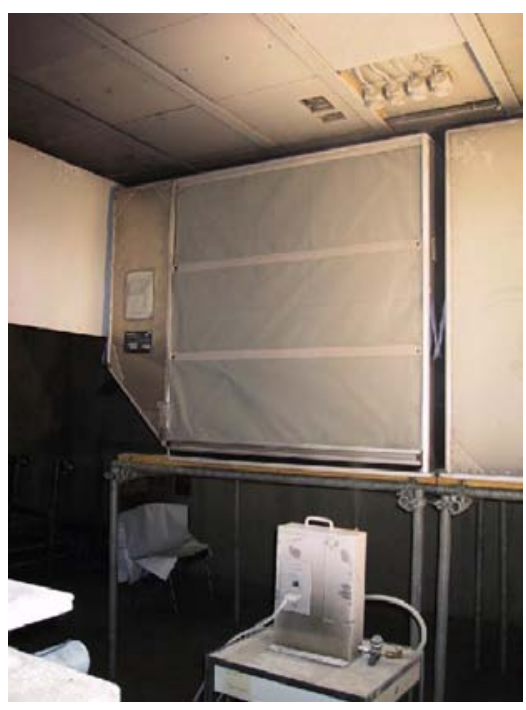

Fig. 11. Set-up for the dust test, bulk bay configuration.

The total amount of dust injected into the test cell was controlled by the on-time of the dust generator, it was used as the key metric for determining a system's immunity to dust. Dust levels in the test cell were measured with a separate particle counter, whose inlet tube was placed in the ceiling of the test cell. The particle counter values were used to verify that the dust generation was valid. In preliminary testing, the dust generator was placed in several locations in the attempt to satisfy two objectives:

- Subject the traditional smoke detectors and the CFVS to the same dust environment.

- Determine the amount of dust generator on-time to trigger a smoke alarm from the traditional smoke detectors. 
The main CFVS dust-immunity requirement is that the CFVS must not indicate a smoke alarm for a given time after the start of dust generation. Figure 12 shows a typical example of CFVS performance compared to actual behaviour of production A340 smoke detectors.

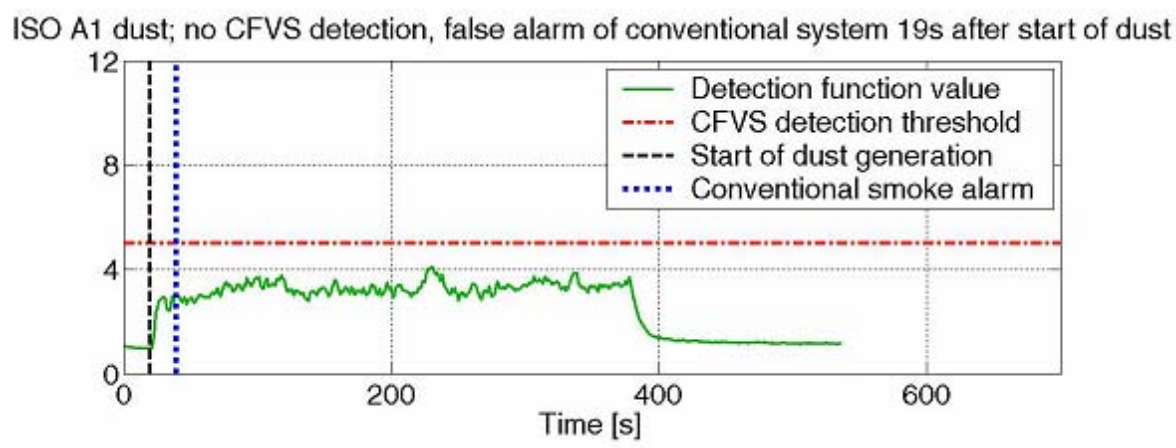

Fig. 12. ISO A1 dust test with a false alarm of a conventional smoke detector

The decision function shown is based on analysis of the overhead view and is used to detect smoke. In the dust test, the CFVS detection threshold is never crossed, while the conventional system issues an alarm only 19 seconds after start of smoke generation. The amount of dust was sufficient to trigger the conventional detectors. In this case the CFVS would unconfirm the smoke alarm as false. This illustrates the improved immunity of the CFVS to common false alarm causes.

\section{CONCLUSIONS}

The CFVS image processing algorithms and their specific parameters were developed based on a large video database, involving smoke, fog, dust and other aerosols. The primary experimental location was in University of Duisburg fire lab. Fire and smoke experiments were also conducted in a cargo bay mock-up in Trauen, Germany, for accurate simulation of a fully loaded cargo bay.

Fog and smoke data in simulated take-off conditions were collected in a National Technical Systems pressure chamber in Boxborough, Massachusetts, USA. Finally, smoke experiments were also conducted on the ground in an A340 aircraft made available by Airbus. The collected database was used to define the exact image features, filtering constants and detection threshold values to be used in the CFVS software. While the concept of video-based detection is well known and was practically used in buildings or tunnels [12], the CFVS is the first vision system suitable for actual deployment in a commercial aircraft. In performance tests, the CFVS detected all fire cases 20 to 350 seconds before the conventional A340 smoke detection system. At present, the CFVS camera and overhead illumination units are fully developed and packaged, meeting all Airbus and FAA/JAA requirements. The control unit along with its software is also fully developed. As such the CFVS is fully certifiable. Although it was developed with Airbus A340 in mind, it may be modified for other aircraft as well.

The advantage of the CFVS over conventional smoke detection is twofold. Firstly, it is able to detect flames and hotspots directly from NIR images. Therefore, it may detect low-smoke fires much faster than traditional smoke detectors. Secondly, the system makes use of the distributed nature of video information. Instead of a small number of 
discrete points, a camera image integrates data from a large volume, which enables earlier detection. Use of switched lighting allows analysis of different features of smoke and non-smoke aerosols, thereby providing means of fire/non-fire differentiation absent in conventional detectors. The result is a detection system that meets or exceeds all fire detection requirements with greatly improved false alarm immunity. In performed tests, CFVS detection algorithms were able to differentiate between all dust and smoke cases, and between majority of fog and smoke cases, but provided correct detection of all cases involving smoke produced by test fires. It reduced fog-related false alarms by more than $50 \%$ and showed almost perfect immunity to dust levels that tripped the conventional smoke detection system.

The existing testing guidelines and standards for qualification of current smoke detection systems were formulated mainly to address the case of traditional detection technologies. It was necessary to develop a suite of fire sensitivity tests, non-fire tests with dust and test room modifications applicable to vision based fire detection systems. Modified tests with room modifications in the Duisburg fire lab show good match with tests performed in a cargo mock-up.

\section{REFERENCES}

[1] Schmötzer, K., "Aircraft Fire Detection: Requirements, Qualification and Certification Aspects," in Proceedings of $12^{\text {th }}$ International Conference on Automatic Fire Detection "AUBE '01", pp. 630-640, March 2001.

[2] JAR/FAR 25.858, Requirements for Fire Detection Systems.

[3] Blake, D., "Initial Development of Improved Aircraft Compartment Fire Detection Certification Criteria," in Proceedings of $12^{\text {th }}$ International Conference on Automatic Fire Detection "AUBE '01", pp. 615-629, March 2001.

[4] Mangon, P., "Fire Detection for Aircraft Cargo Compartments, Reduction of False Alarms," in Proceedings of $12^{\text {th }}$ International Conference on Automatic Fire Detection "AUBE '01", pp. 653-664, March 2001.

[5] Sadok, M., Zakrzewski, R., and Zeliff, B., "Video-based cargo fire verification system with fuzzy inference engine for commercial aircraft," IS\&T/SPIE 17th Annual Symposium on Electronic Imaging, Proc. SPIE Vol. 5679, pp. 99-107, Jan. 2005.

[6] Gonzalez, R.C. and Woods, R.E., Digital Image Processing, Prentice Hall, 2002.

[7] EN-54, Part 9, Fire Sensitivity Tests, BSi Standards, 1984.

[8] CEA, GEI 1-049, Test Methods for Multisensor Smoke Detectors, 1997.

[9] Shirer, J., “Test Fire and Dust Threshold Update Summary,” Goodrich Internal Report, October 2003.

[10] ISO 4225:1994, Air Quality - General Aspects - Vocabulary, 1994.

[11] ISO 12103-1:1997, Road Vehicles - Test Dust for Filter Evaluation - Arizona Test Dust, 1997.

[12] Wieser, D. and Brupbacher, T. "Smoke Detection in Tunnels Using Video Images," in Proceedings of $12^{\text {th }}$ International Conference on Automatic Fire Detection "AUBE '01", pp. 79-90, March 2001. 